Communications in Physics, Vol.21, No. 1 (2011), pp. 89-96

\title{
INVESTIGATION OF 1D PHOTONIC CRYSTAL BASED ON NANO-POROUS SILICON MULTILAYER FOR OPTICAL FILTERING
}

\author{
DO THUY CHI \\ Thai Nguyen College of Education, Thai Nguyen University \\ BUI HUY AND NGUYEN THUY VAN \\ Institute of Materials Science, VAST \\ PHAM VAN HOI \\ Institute of Materials Science, VAST \\ and \\ University of Engineering and Technology, Hanoi National University
}

\begin{abstract}
We present the fabrication, simulation, and measurements of $1 D$ photonic crystal based on nano-porous silicon multilayer designed as an optical interference filter. Using electrochemical etching with timely repeat steps of applied current densities, we fabricated a multilayer structure composed of alternating high-and low-index layer which achieved $90 \%$ power reflectivity at wavelength range of 1400-3000 $\mathrm{nm}$. The simulation is relying on the Transfer Matrix Method (TMM) to design and predict the optical properties of nano-porous silicon multilayer as well as the relation between anodization parameters with reflection spectra. The measured reflection and transmission spectra of the nano-porous silicon multilayer show good agreement with simulation. This technique could provide a convenient and economical method to produce filters, cavities, and graded-index dielectric waveguides in the future.
\end{abstract}

\section{INTRODUCTION}

Several techniques have been used to fabricate optical-band multilayer filters, including stacking and assembly of semiconductor, ceramics, and plastic films [1-3] and other dielectrics. Thick film sputter-deposition method has also been used to fabricate multilayer of silicon, oxides and other semiconductors [4]. Polymer nanoparticle composites have been used to provide additional flexibility in controlling the effective refractive index of layers [5]. Nano-porous silicon (nano-PS) is an easily fabricated nanoscale composite of air and silicon that has been shown to be suitable for optical filters [6]. The considerable and controllable variations in refractive index of nano-PS fabricated by an electro-chemical etching make it become a promising material as photonic crystal. In the porous structure, PS consists of many pores and silicon residuals and, usually, can be described as a homogeneous mixture of silicon, air and, eventually, silicon dioxide. Based on porosity, PS can be classified into three types: nano-, meso- and macro-pores. In the case of PS nano- and meso-pores, the size of both the silicon residuals and the air voids (pores) can be in the range of few nanometers to tens ones. Since infrared light has wavelengths of micrometers, 
PS can be optically described in the infrared range as an "effective medium" [7,8], whose optical properties mainly depend on the relative content of silicon and air (i.e. porosity). The one-dimension (1D) photonic crystal based on nano-PS multilayer is possible because [9]: (i) the etching process is self-limited (i.e. once porous layer is formed, the electrochemical etching of this layer stops); (ii) the etching occurs mainly in correspondence of the pore tips; (iii) the porosity depends only upon the current density once the other etching parameters kept fixed; and (iv) the refractive index of nano-PS depends on its porosity. Therefore, by varying the current density during the etching process; it is possible to vary porosity in the etching direction. By this way, the forming a stack of nano-PS layers of different porosities (and hence, different refractive index) results in a dielectric multilayer. Dependence on structure as well as number of layers, the stack can act as an optical interference filter [10,11], waveguide [12], omni-directional mirror [13], rugate filter [14], and microcavity [15]. In those optical devices, the interference filter may be basic for other ones. Optical interference filter is narrow-band dielectric mirror obtained by stacking periodically two layers of a high $(\mathrm{nH})$ and low $(\mathrm{nL})$ refractive-index material whose thickness is $\lambda / 4 \mathrm{n}$, where $\lambda$ is the wavelength, at which the reflectance would be maximal. Compared with other methods, the electro-chemical etching method avoids the difficulty associated with stacking and assembly of dielectric layers, eliminates the need for lengthy deposition of thick films, and permits a wide range of refractive indices to be fabricated from a single silicon substrate [16].

In this work, we investigate the 1D photonic crystals based on nano-porous silicon multilayer designed as an optical filter. Before fabricating the filters, a simulation program based on the Transfer Matrix Method (TMM) [17] has been set up in order to design and predict the optical properties of nano-PS multilayer. The elaborated filters have the wavelength selective properties in the range from 1400 to $3000 \mathrm{~nm}$, and reflectivity is about $90 \%$. The spectral and structural characteristics of the filters have been compared with simulation ones.

\section{EXPERIMENTAL SETUP}

The porous silicon multilayer was fabricated using electro-chemical etching of p-type (100) silicon wafers with resistivity of $0.01-0.1 \Omega$.cm in $13 \%-20 \%$ hydrofluoric acid (HF): ethanol solution. The electro-chemical process was carried out without illumination. The process was computer-controlled galvanostat, so precise control over current density and etching time was achieved, then it is resulting in a good control of the refractive index and thickness over the individual layers forming the multilayer. We had fabricated and characterized single-layer samples with low and high porosities in our previous work [18]. Multilayer was formed by periodically varying the applied current density with two levels $\left(J_{1}\right.$ and $\left.J_{2}\right)$ between 19 and $64 \mathrm{~mA} / \mathrm{cm}^{2}$ as presented in Fig. 1. The number of layers for each filter is from 12 to 36 . The multilayer has been rinsed in methanol and isopropanol after anodization and dried with nitrogen gas. The macro- and micro-structural characteristics of nano-PS multilayer were carried out in an Ultrahigh Resolution Field-Emission Scanning Electron Microscopy (FE-SEM) S-4800 and the reflectance spectra of filters were studied by UV - VIS - NIR spectrophotometer Varian Cary 5000. 


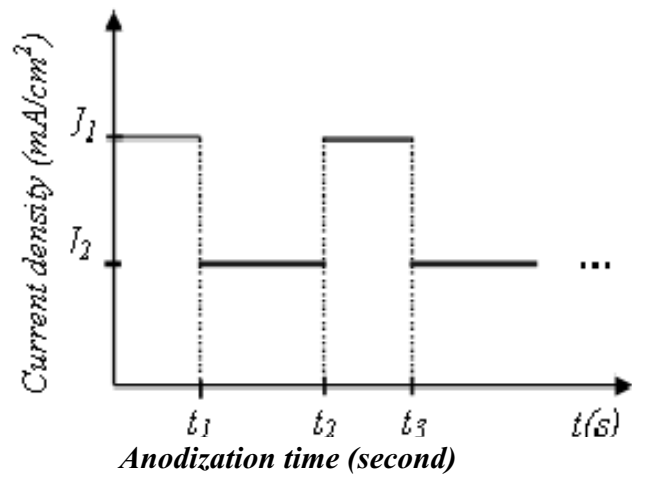

Fig. 1. Current density versus anodization time

\section{RESULTS AND DISCUSSIONS}

In this section, firstly, we present the simulation program based on the Transfer Matrix Method (TMM) for the analysis of optical interference filters. Then, we compare the simulated optical responses with that of fabricated filters.

\section{Simulation Results}

A simulation program based on the TMM has been realized on wave transmission in one-dimensional structure. We consider a 1D structure consisting of alternating nano-PS layers of different refractive indices coupled to a homogeneous medium characterized by refractive index $n_{0}$ at the initial medium and ns at final medium. The schematic of 1D multilayer structure is shown in Fig. 2. The $n_{1}$ and $n_{2}$ are the layers refractive indices with the thicknesses of $h_{1}$ and $h_{2}$, respectively. The $\lambda$ is called the period of $1 \mathrm{D}$ structure $\left(\Lambda=h_{1}+h_{2}\right) \dot{A}(x)$ represents the amplitude of the right-traveling-wave and $B(x)$ that of the left-traveling one. If the amplitude of the plane waves at interface $x=x_{m}$ (see Fig. 2) $A_{m}$ and $B_{m}$ is presented as vectors, the relation between $A_{0}$ and $B_{0}$ and $A_{S}^{\prime}, B_{S}^{\prime}$ can thus be written as [19]:

$$
\left(\begin{array}{c}
A_{0} \\
B_{0}
\end{array}\right)=D_{0}^{-1}\left[D_{1} P_{1} D_{1}^{-1} D_{2} P_{2} D_{2}^{-1} \ldots\right]^{N} D_{S}=\left(\begin{array}{cc}
M_{11} & M_{12} \\
M_{21} & M_{22}
\end{array}\right)\left(\begin{array}{c}
A_{S}^{\prime} \\
B_{S}^{\prime}
\end{array}\right)
$$

Where $N$ is the number of layers in the structure. The dynamical matrix Dm is written by:

$$
D_{m}=\left\{\begin{array}{l}
\left(\begin{array}{cc}
1 & 1 \\
n_{m} \cos \theta_{m} & -n_{m} \cos \theta_{m}
\end{array}\right) \quad \text { for TE wave } \\
\left(\begin{array}{cc}
\cos \theta_{m} & \cos \theta_{m} \\
n_{m} & -n_{m}
\end{array}\right) \quad \text { for TM wave }
\end{array}\right.
$$

and the propagation matrix Pm can be written as:

$$
P_{m}=\left(\begin{array}{cc}
e^{i k_{m x} h_{m}} & 0 \\
0 & e^{-i k_{m x} h_{m}}
\end{array}\right)
$$


Where $k_{m x}=\Omega n_{m} \cos \theta_{m}$ is the $x$ component of the wave vector and $\theta_{m}$ is the ray angle in each layer. If the light is incident from lossless medium n0, the reflectance $\mathrm{R}$ can be calculated as:

$$
R=\left|\frac{M_{21}}{M_{11}}\right|^{2}
$$

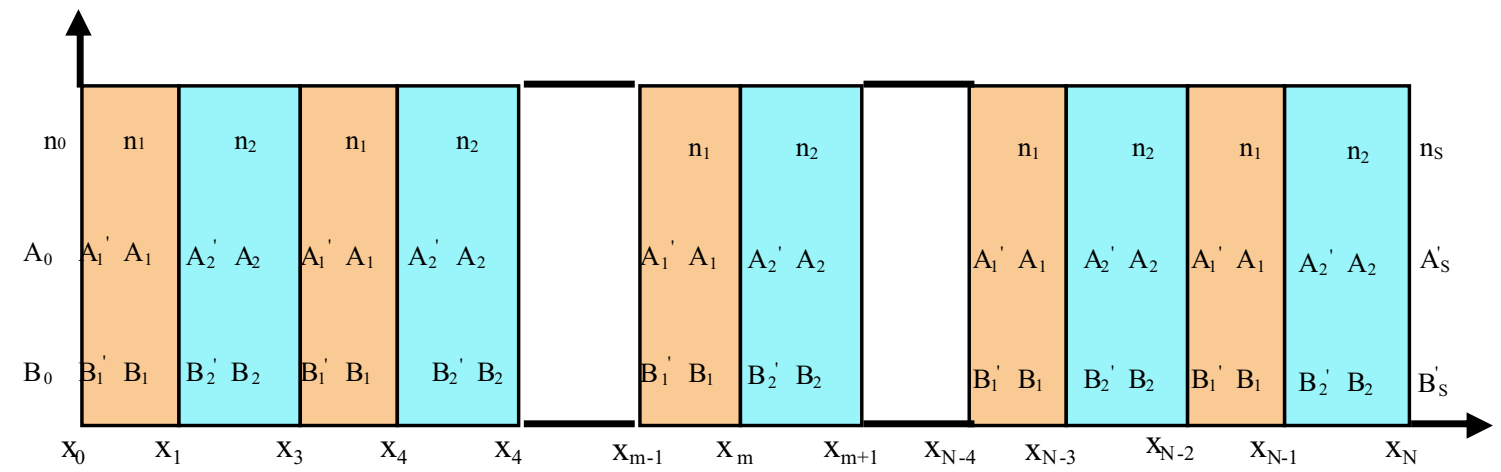

Fig. 2. Structural schematic of $1 \mathrm{D}$ photonic crystal based on multilayer system

Based on above-mentioned theory, we set up a program for the simulation of multilayer structure by using Matlab software. This program contains the following parameters: (i) Refractive index of ambient medium $\left(n_{0}\right)$; (ii) Refractive index of substrate $\left(n_{s}\right)$; (iii) Incidence angle $(\theta)$; (iv) Number of layers (N) into the 1D nano-PS multilayers; (v) Refractive indices $\left(n_{m}\right)$; (vi) thickness $\left(h_{m}\right)$ of periodic layers and (vii) wavelength $\lambda$ varied from the initial to the final values of reflective wavelength.

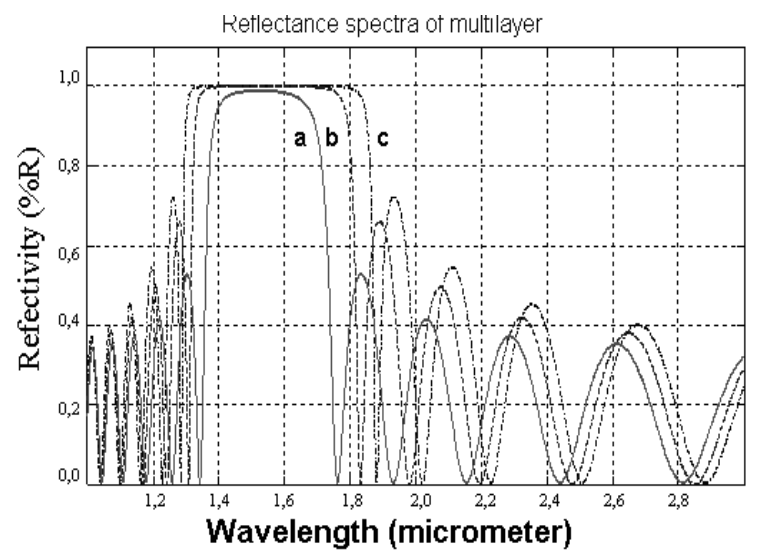

Fig. 3. Reflectivity spectra versus of $n_{1} / n_{2}$ ratio. Curves a, b, and c, corresponding to ratio $2.0 / 1.5,2.2 / 1.5$ and $2.5 / 1.5$

Figure 3 shows the calculated reflective spectra of three filters with 24-layer samples and the thickness of one layer has been calculated to obtain a centered reflection wavelength 
at $1550 \mathrm{~nm}$. The parameters for simulation are: thickness layers $h_{1}=\lambda / 4 . n_{1} ; h_{2}=\lambda / 4 . n_{2}$, $n_{0}=1$ and $n_{s}=3.5$. The calculated values of refractive indices of multilayers $n_{1}, n_{2}$ had been choice from 1.5 to 2.5 , which is suitable with experiment. Calculation shows that the ratio of $n_{1} / n_{2}$ is strongly influenced on the line-width and sharpness of the spectra. The increase of $n_{1} / n_{2}$ leads to the spectral broadening. The influence of the number of layers $(N)$ on the reflection spectra has been shown in Figure 4 . When $N$ increases, the spectra are sharper and the reflectivity tends to the unity.

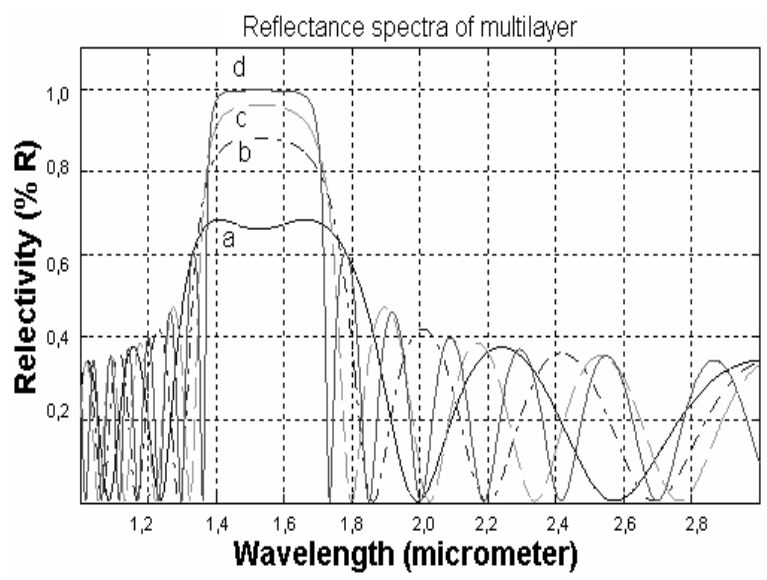

Fig. 4. Reflectivity spectra versus number of periods. Curves a, b, c and d, corresponding layer numbers of $12,16,20$, and 30 , respectively

\section{Experimental results}

The nano- and micro-structures of the 1D photonic crystals have been analyzed by Field-Emission Scanning Electron Microscopy (FE-SEM). Figure 5 is a SEM-image of the completed nano-porous silicon multilayer fabricated by ratio of current densities $J_{1} / J_{2}=64 / 19$ and duration time of 6.33 and 12.3 seconds, respectively. As seen in Figure $5 \mathrm{a}$, the typical sizes of the silicon residuals and air voices are about $50 \mathrm{~nm}$. The SEMimage of multilayer shows different gray levels depending on the porosity of the layers (see Fig. 5b). Based on this difference, the layers of the stack are distinguished and therefore the thickness of each layer can be experimentally determined. In our case thickness of each layer was measured of about $250 \mathrm{~nm}$, which is designed center wavelength of $1550 \mathrm{~nm}$. Figure 6 present the reflective spectra of multilayer samples with different thickness. The curves (a) and (b) show spectra of 24-layer filters, which have total thickness of $4.4 \mu \mathrm{m}$ and of $6.0 \mu \mathrm{m}$ fabricated at a ratio of current densities $J_{1} / J_{2}=64 / 19$ and $38 / 19$, respectively. The difference in thickness of completed multilayer filters and therefore thickness of the layer depended on the duration time and current density of electro-chemical process. The change of thickness of the stacks causes the shift of the center wavelength of reflectance by the Bragg law.

When the duration time of electro-chemical etching process was kept constantly and the current density would be changed, the samples have the same wavelength $(\lambda)$ at 


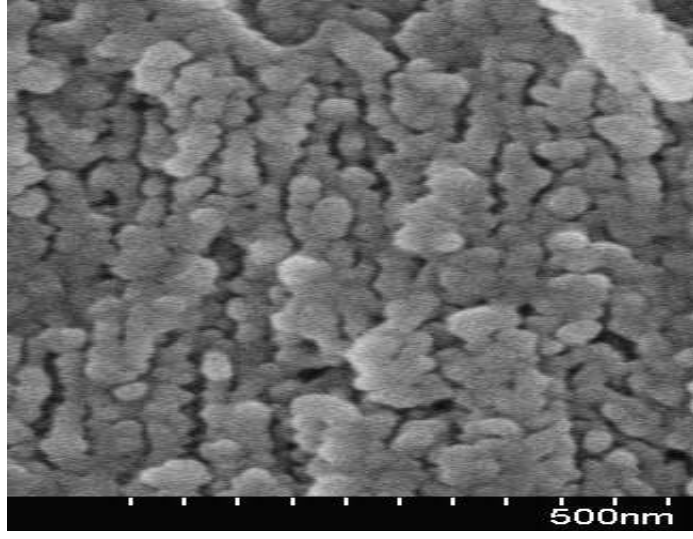

a

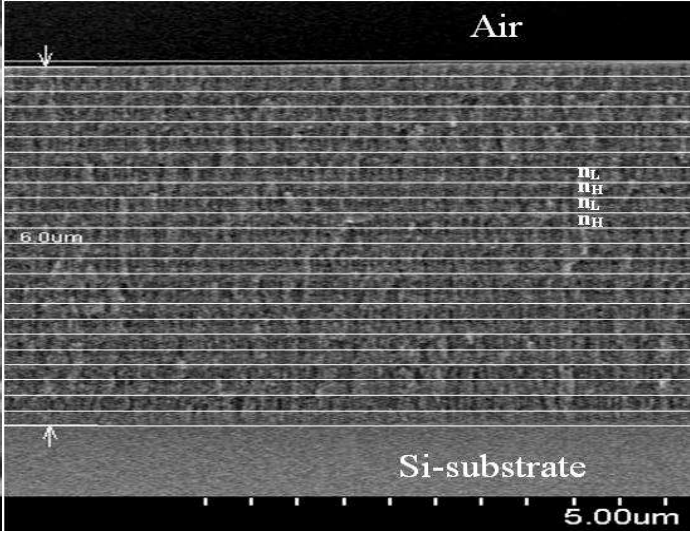

b

Fig. 5. Cross-sectional SEM images of micro- (a) and macro-(b) structure from a filter with $N=12$

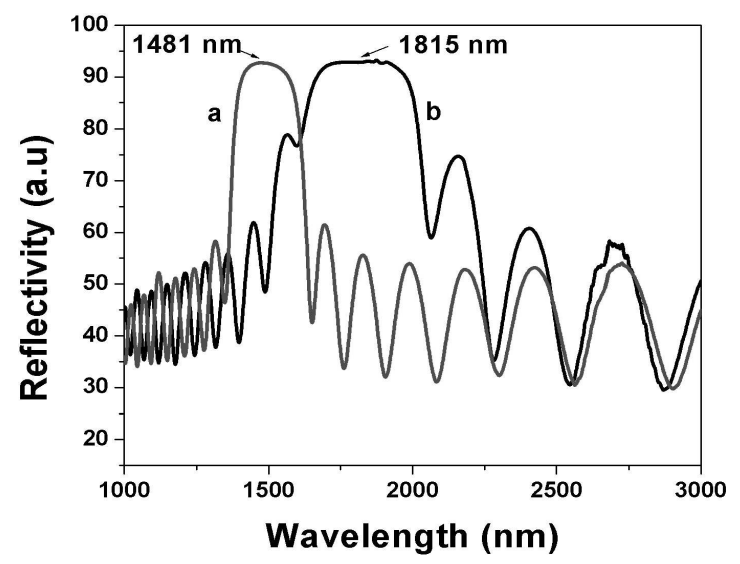

Fig. 6. Shifting the reflectance spectra by varying a thickness of the 24-layers in the stacks. (a) $d=4.4 \mu \mathrm{m}$; (b) $d=6.0 \mu \mathrm{m}$

maximal reflectance. In this case the line-width of reflective spectra was slightly broadened with increasing current density. This experimental result exhibit good agreement with calculation.

Figure 7 shows reflective spectra from samples having different number of layers. As seen in this figure, when the number of layers $\mathrm{N}$ increases the spectra become shaper and the reflectivity is increasing. The difference in the shape of the reflective spectra from simulation and experiment occurs in the filters having few layers (for $N \leq 12$ ). In this case, the reflection from the interfaces between air and sample becomes more important, so that the imperfection of interfaces created by the electro-chemical etching can cause a deformation of reflective spectrum. 


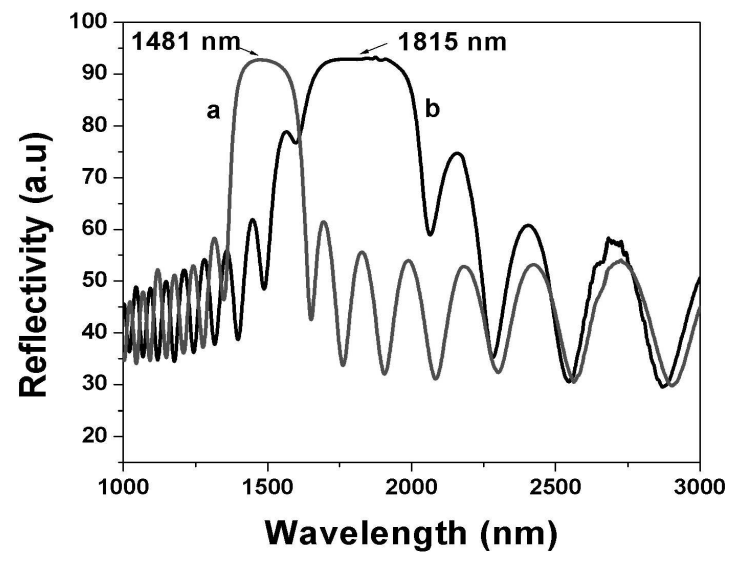

Fig. 7. Reflective spectra vs. $N$. Curves a, b and c for filters with $N$ of 12,36 and 24 , respectively

In the case of filters having too few layers $(N \leq 12)$, reflection from the interfaces, especially from the interface between air and the top layer, becomes more important in those filters, so that the imperfections of interfaces created by electro-chemical etching can cause a deformation of the reflective spectrum as seen in curve (a) of Figure 7 . In the case of the filters having too many layers $(N \geq 36)$, the long anodisation time causes a deformation of the nano-structure of surface layers whereas the nano- and micro-structure of the bottom layers can be affected by the slower transport of etching substances. All of these factors lead to a break in condition of the Bragg reflection in such layers and the decrease in reflectivity of the filter as shown in curve (b) of Figure 7.

\section{CONCLUSSIONS}

To conclude, using the electro-chemical etching process we successfully fabricated the $1 \mathrm{D}$ photonic crystals based on nano-porous silicon multilayer. The multilayer samples have the selectivity of wavelength in a range from $1400 \mathrm{~nm}$ to $3000 \mathrm{~nm}$, and the reflectivity of about $90 \%$. The spectral characteristics of those 1D photonic crystals such as desired wavelength $\lambda$, the FWHM of spectrum, and reflectance have been controlled. Simulation programs have been realized for the mathematical study and design of filters and its result was an excellent agreement with experimental one. The imperfection of interfaces created by electro-chemical etching may be caused the deformation of reflective spectrum from filters having few layers. These results show that the Bragg grating filter used only two different porosities, in principle one could construct arbitrary refractive index profile by adjusting the current density of electro-chemical etching process as a function of time. This method can use for making more complex filters, cavities, and graded-index dielectric waveguides. 


\section{ACKNOWLEDGEMENTS}

This work is financially supported by National Foundation for Science and Technology Development (NAFOSTED) of Vietnam under Grant No.103.06.38.09. The authors

thank Pham Duy Long for supplying the Electro-chemical equipment. This work had been using the apparatus of National Key Laboratory for Electronic Materials and Devices.

\section{REFERENCES}

[1] R. Schiwon, G. Schwaab, E. Brundermann, M. Havenith, Appl. Phys. Lett. 83 (2003) 4119

[2] N. Matsumoto, T. Nakagawa, K. Kageyama, N. Wada, and Y. Sakabe, Jpn. I. Appl. Phys. Part1 45 (2006) 7499

[3] D. Turchinovich, A. Kammoun, P. Knobloch, T. Dobbertin, and M. Koch, Appl. Phys. A 74 (2002) 291

[4] I. Hosako, Appl.Opt. 44 (2005) 3769

[5] J. Lott, C. Xia, L. Kosnosky, C. Weder, J.Shan, Adv. Mater. 20 (2005) 3649

[6] P. Bettotti, M. Cazzanelli, L. Dal Negro, B. Danese, Z. Gaburro, C. J. Oton, G. Vijaya Prakash, and L. Pavesi, J. Phys.: Condens. Mater. 14 (2002) 8253

[7] W. Thei, Surf. Sci. Rep. 29 (1997) 95

[8] Bui Huy, Pham Van Hoi, Phan Hong Khoi, Do Khanh Van, Pham Thanh Binh, Tran Thi Cham, J. of Phys.: Conf. series 187 (2009) 012033

[9] C. Mazzoleni and L. Pavesi, Appl. Phys. Lett. 67 (1995) 2983

[10] M. G. Beger, R. Arens-Fischer, M. Thonissen, M. Kruger, S. Billat, H. Luth, S. Hilbrich, W. Thei, P. Grosse, Thin Sol. Films 297 (1977) 237

[11] D. Hunkel, R. Buts, R. Ares-Fisher, M. Marso, H. Luth, J. Luminescence 80 (1999) 133

[12] Andrea M. Rossi, Giampiero Amato, Vittorio Camarchia, Luca Boarino, and Stefano Borini, Appl. Phys. Lett. 78 (2001) 3003

[13] W. H. Zheng, P. Reece, B. Q. Sun, and M. Gal, Appl. Phys. Lett. 84 (2004) 3519

[14] S. Ilyas, T. Bocking, K. Kilian, P. J. Reece, J. Gooding, K. Gaus, and M. Gal, Opt. Mater. 29 (2007) 619

[15] V. Mulloni, C. Mazzoleni and L. Pavesi, Semicond. Sci. Technol. 14, (1999) 1052

[16] Shu-Zee A. Lo and Thomas E. Murphy, Opt. Lett. 34 (2009) 2921

[17] M. Born and E. Wolf, Principles of Optics, Chapter 3, University Press, Cambridge, UK, 7th Edition, 1999

[18] Bui Huy, Tran Thi Cham, Ha Xuan Vinh, Do Khanh Van, Pham Van Hoi, J. Korean Phys. Soc. 53 (3) (2008) 1397

[19] C. Zhang, F. Qiao, J.Wan, and J.Zi, J. Appl. Phys. 87 (2000) 3174

Received 09 October 2010. 